\title{
INTERAÇÃO ENTRE DENSIDADE DE SEMEADURA E DIFERENTES INOCULANTES NO DESEMPENHO SIMBIÓTICO E AGRONÔMICO DA SOJA
}

\author{
BARBARO-TORNELI, Ivana Marino ${ }^{1}$; SANTOS, Guilherme Xavier Lúcioº ${ }^{2}$ MARTINS, Monica \\ Helena $^{2}$; CORDEIRO-JUNIOR, Paulo Sérgio ${ }^{3}$; CORREA, Alessandra Neves ${ }^{2}$; POLLI, Kátia Cristina ${ }^{4}$; \\ FINOTO, Everton Luis ${ }^{5}$; DONÁ, Sérgio ${ }^{6}$; NAKAYAMA, Fernando Takayuki ${ }^{7}$; SOARES, Maria Beatriz \\ Bernardes $^{8}$
}

ISSUE DOI: $10.3738 / 1982.2278 .3631$

\begin{abstract}
RESUMO: O arranjo espacial de plantas é alterado pela densidade de semeadura e pelo espaçamento entre as fileiras. Por outro lado, em soja, a fixação biológica de nitrogênio (FBN) com uso de inoculantes é a principal forma de disponibilizar o nitrogênio para a planta. Para o Estado de São Paulo, principalmente em áreas de renovação de canavial têm se observado a necessidade de maiores esclarecimentos em relação a essas questões. Assim, objetivouse avaliar o comportamento simbiótico e agronômico de soja submetida a três tratamentos: inoculação, controle e coinoculação no sulco de semeadura nas densidades de semeadura: 14,16 e 18 sementes $\mathrm{m}^{-1}$. O experimento foi instalado em esquema de parcelões demonstrativos em semeadura direta na palhada de cana-de-açúcar em TanabiSP, safra 2018-19. A cada nove linhas da semeadora-adubadora foi inserida uma densidade de semeadura. Os tratamentos inoculação, controle e coinoculação foram dispostos a cada $50 \mathrm{~m}$ de comprimento. O delineamento experimental utilizado foi o de faixas 'Split-Block", com três repetições. Em $\mathrm{R}_{1}$, avaliaram-se alguns parâmetros de FBN e em $\mathrm{R}_{8}$, caracteres agronômicos e produtividade de grãos. Os dados obtidos foram submetidos a análise de variância pelo teste $\mathrm{F}$ e as médias comparadas pelo teste de Tukey a 5\%. Constatou-se diferenças expressivas na interação de densidade de semeadura versus tratamentos para várias características avaliadas. Na densidade de 16 sementes $\mathrm{m}^{-1}$ a inoculação no sulco de semeadura destacou-se incrementando a produtividade de grãos em 6,61 sacas $\mathrm{ha}^{-1}$ a mais quando comparada a coinoculação no sulco.
\end{abstract}

Palavras-chave: Fixação biológica de nitrogênio. Glycine max (L). População de plantas. Rendimento de grãos.

\section{INTERACTION BETWEEN DENSITY OF SOWING AND DIFFERENT INOCULANTS IN SOYBEAN SYMBIOTIC AND AGRONOMIC PERFORMANCE}

SUMMARY: The spatial arrangement of plants is altered by seeding density and spacing between rows. On the other hand, in soybean, the biological nitrogen fixation (BNF) with the use of inoculants is the main way of making nitrogen available to the plant. For the State of São Paulo, especially in areas of sugarcane renewal, the need for further clarification regarding these issues has been observed. The objective of this study was to evaluate the symbiotic and agronomic behavior of soybeans submitted to three treatments: inoculation, control and co-inoculation in the sowing furrow at sowing densities: 14,16 and $18 \mathrm{~m}^{-1}$ seeds. The experiment was installed in a demonstration plots scheme in no-tillage in the sugarcane straw in Tanabi-SP, harvest 2018-19. A seeding density was inserted into each row of the seed drill. The inoculation, control and co-inoculation treatments were arranged every $50 \mathrm{~m}$ in length. The experimental design used was 'Split-Block', with three replications. In R1, some parameters of FBN and $\mathrm{R} 8$, agronomic traits and grain yield were evaluated. The data were submitted to analysis of variance by the $\mathrm{F}$ test

\footnotetext{
${ }^{1}$ Pesquisadora Científica, Dra. Agência Paulista de Tecnologia dos Agronegócios-APTA/ Secretaria de Agricultura e Abastecimento - SAA - Polo Regional Alta Mogiana - Colina, SP;

${ }^{2}$ Engenheiro Agrônomo, Bolsista de Aperfeiçoamento Técnico FUNDAG/APTA/SAA - Polo Regional Centro Norte - Pindorama;

${ }^{3}$ Eng. Agrônomo, Mestrando em Produção Vegetal, FCAV-UNESP- Jaboticabal,SP;

${ }^{4}$ Graduanda em Agronomia na UNIRP, S. J. do Rio Preto, SP, Bolsista PIBIC/CNPq/APTA/SAA - Polo Regional Centro Norte - Pindorama;

${ }^{5}$ Eng. Agrônomo, Dr., Pesquisador Cientifico na APTA/SAA-Polo Regional Centro Norte - Pindorama;

${ }^{6}$ Eng. Agrônomo, Msc., Pesquisador Cientifico APTA/SAA-Polo Regional Médio Paranapanema - Assis;

${ }^{7}$ Eng. Agrônomo, Dr., Pesquisador Cientifico na APTA/SAA-Polo Regional Alta Paulista - Adamantina.

${ }^{8}$ Eng. Agrônomo, Msc., Pesquisador Cientifico na APTA/SAA - Polo Regional Centro Norte - Pindorama;
} 
and the means were compared by the Tukey test at 5\%. It was verified significant differences in the interaction of sowing density versus treatments for several evaluated characteristics. In the density of $16 \mathrm{~m}^{-1}$ seeds inoculation in the sowing groove was highlighted increasing the yield of grains in $6.61 \mathrm{bags} \mathrm{ha}^{-1}$ more when compared to the coinoculation

Keywords: Biological fixation of nitrogen. Glycine $\max (\mathrm{L})$. Plants population. Yield grains

\section{INTRODUÇÃO}

O Estado de São Paulo apesar da pouca expressividade em termos de área cultivada (962 mil hectares em 2016/17) e produção de soja quando comparado aos principais estados produtores Mato Grosso, Paraná, Rio Grande do Sul e Goiás, respectivamente, os quais representam 69\% da produção total de soja do país (CONAB, 2018), depara na reforma de canaviais uma oportunidade para expansão da área cultivada, visto que anualmente são disponibilizados ao redor de 722.294 ha (CANASAT, 2016).

$\mathrm{O}$ arranjo espacial de plantas é alterado pela densidade de semeadura e pelo espaçamento entre as fileiras, sendo que a mudança desses fatores pode proporcionar aumentos na produtividade (SOUZA et al., 2010; PROCÓPIO et al., 2013), sem grandes impactos no custo de produção. Além da produtividade, a densidade de semeadura pode alterar a velocidade de fechamento das entrelinhas, a incidência de plantas daninhas, insetos-praga e doenças, a penetração de agroquímicos no dossel, o acamamento das plantas e a qualidade dos grãos colhidos.

Sendo assim, para que sejam obtidas diferentes densidades de semeadura, e por conseguinte, populações de plantas, são necessárias variações na regulagem da semeadora-adubadora (CANOVA et al., 2007). Por outro lado, a falta de resposta diferenciada no rendimento dos grãos a variação da densidade de semeadura da soja está intimamente relacionada a plasticidade fenotípica que esta cultura apresenta (RAMBO et al., 2003).

Santos et al. (2018) relatam que o comportamento de cultivares de soja sob diferentes condições de cultivo se torna fundamental na busca do entendimento do manejo da cultura. Desta forma, ainda segundo esses autores, a densidade de semeadura é prática que devem ser aprimoradas para maior eficiência do sistema, sendo assim, a identificação de um número de plantas que resulte em uma competição intraespecífica que permita um melhor aproveitamento dos recursos disponíveis para o crescimento e rendimento de grãos é imprescindível.

Outra questão muito importante no mundo e no Brasil refere-se na substituição ou minimização do uso de insumos minerais industrializados por organismos biológicos a fim de reduzir a contaminação do ambiente dada a crescente busca por maior sustentabilidade do sistema agrícola, além da crescente demanda de alimentos saudáveis. Por isso, um dos grandes desafios da pesquisa é o desenvolvimento de técnicas de manejo que visam ao uso dos fatores biológicos no incremento da produção (ZUFFO, 2016).

No que tange a soja, a principal fonte de nitrogênio é obtida por meio da fixação biológica de nitrogênio (FBN) que consiste em um processo biológico na qual o nitrogênio abundante presente na atmosfera $\left(\mathrm{N}_{2}\right)$ é transformado em formas assimiláveis pela planta: amônio (NH4+) ou nitrato (NO3-), por meio de bactérias (conhecidas de modo coletivo por rizóbios). Desta forma, o insumo inoculante é obtido pelo sojicultor a muito baixo custo no mercado, proporcionando a cultura da soja a não dependência do uso de fertilizante mineral nitrogenado.

Ainda nesse cenário, a prática de coinoculação validada a apenas cinco anos no Brasil, tem atuado como coadjuvante no processo pois promove melhoras no processo já tão eficiente da FBN em soja proporcionando consequentemente acréscimos nos patamares de produtividade da cultura. Esta técnica é 
muito simples, uma vez que utiliza a inoculação mista de dois gêneros de bactérias: o Bradyrhizobium japonicum e elkkanie até então utilizados na inoculação tradicional de soja, com o uso da bactéria Azospirillum brasilense, uma bactéria diazotrófica de vida livre que pode se associar ao sistema radicular das plantas e que vem sendo utilizada para inoculação em gramíneas como: milho e trigo associada a doses reduzidas de fertilizantes sintéticos nitrogenados (FERLINI, 2006; BÁRBARO et al., 2008; BÁRBARO et al., 2009; BÁRBARO et al., 2011).

Com base nesse contexto o presente trabalho teve por objetivo avaliar o comportamento simbiótico e agronômico da cultivar de soja BRS 7380 RR semeada diretamente na palhada de cana-deaçúcar e submetida a três tratamentos: inoculação no sulco, controle e coinoculação no sulco de semeadura em três diferentes densidades de semeadura: 14,16 e 18 sementes $\mathrm{m}^{-1}$.

\section{MATERIAL E MÉTODO}

O experimento foi instalado em condições de campo, em propriedade particular no dia 01 de novembro de 2018, em área de renovação de canavial, localizada no município de Tanabi-SP, a $20^{\circ} 37^{\prime}$ 35" S, 49³ 38' 57" W e altitude de 518 m. Segundo a classificação climática proposta por Köppen (2001), tendo como base a temperatura e precipitação, o município de Tanabi está localizado na região caracterizada pelo tipo climático tropical úmido com inverno seco, designado Cwa, com temperaturas médias no mês mais quente acima de $22^{\circ} \mathrm{C}$ e no mês mais frio acima de $18^{\circ} \mathrm{C}$, com duas estações bem definidas, uma chuvosa e quente, de setembro a março, e outra seca e menos quente, de abril a agosto.

O delineamento experimental utilizado foi o de faixas "Split Block" com três repetições. As fontes de variação da análise de variância foram compostas por três densidades de semeadura $(14,16$ e 18 sementes $\mathrm{m}^{-1}$ ) e dos tratamentos: inoculação no sulco de semeadura, controle sem inoculação e coinoculação também no sulco de semeadura. É importante ressaltar que foi realizado o ajuste da densidade de semeadura para as parcelas de acordo com a porcentagem de germinação fornecida pelo fabricante.

Para a implantação do experimento foi utilizada semeadora-adubadora de 9 linhas para cada densidade de semeadura ajustada previamente na semeadora adubadora com espaçamento entre linhas de $0,5 \mathrm{~m}$, sendo a cultivar utilizada a BRS $7380 \mathrm{RR}$. A cada $50 \mathrm{~m}$ para cada tiro da semeadora-adubadora foram distribuídos os tratamentos de: coinoculação no sulco, controle e inoculação no sulco.

Quanto a prática de coinoculação foi utilizada formulação da empresa Stoller do Brasil Ltda contendo os dois gêneros de bactérias e que se encontra em fase de registro no MAPA, sendo que a mesma possui Bradyrhizobium japonicum SEMIA 5079 na concentração de $1 \times 10^{9} \mathrm{UFC} \mathrm{mL}^{-1}$ e Azospirillum brasilense (cepas AbV5 e AbV6) na concentração de $1 \times 10^{7} \mathrm{UFC} \mathrm{mL}^{-1}$, na dose recomendada de $0,5 \mathrm{~L}$ ha ${ }^{1}$. O inoculante para inoculação tradicional com Bradyrhizobium no sulco de semeadura utilizado foi o Masterfix $\mathrm{L}^{\circledR}$ Soja na dose $0,8 \mathrm{~L} \mathrm{ha}^{-1}$, ou seja, sete doses a mais que o tratamento de sementes cuja indicação é de 0,1 a 0,2 $\mathrm{L} \mathrm{ha}^{-1}$. No estádio de desenvolvimento V5 (FEHR; CAVINESS, 1977) foram aplicados os micronutrientes cobalto e molibdênio via pulverização foliar, em todos os tratamentos inclusive controle, na dose de $0,1 \mathrm{~L}$ ha ${ }^{-1}$.

A aplicação dos inoculantes para inoculação e coinoculação foi realizada através de pulverizador acoplado a semeadora-adubadora. Foram adotados alguns cuidados para garantir uma maior eficiência dos tratamentos, como preparo da calda a sombra, averiguação do $\mathrm{pH}$ da calda e calibração adequada dos bicos de pulverização para distribuição uniforme dos inoculantes.

Os parâmetros avaliados no início do florescimento, no estádio fenológico R1 foram: número de nódulos totais (NNODT) em número planta ${ }^{-1}$; massa seca nodular total (MSNNOT) em mg planta ${ }^{-1}$; altura 
de planta no florescimento (APF) em $\mathrm{cm}$; massa seca da raiz (MSR) em g.planta ${ }^{-1}$; massa seca da parte aérea (MSPA) em g planta ${ }^{-1}$ e o teor de nitrogênio total na massa da parte aérea (TNPA) foi determinado segundo metodologia descrita por Bataglia et al. (1983).

Por ocasião da colheita foram avaliados no estádio reprodutivo R8 os seguintes caracteres agronômicos: estande final em plantas $\mathrm{m}^{-1}$; altura de planta na maturação em $\mathrm{cm}$ (APM); altura de inserção da primeira vagem em cm (AIV); Massa de mil grãos (MMG) em gramas e produtividade de grãos (PG) em kg ha-1 (valores corrigidos para $13 \%$ de umidade).

Todas as técnicas de cultivo de soja, como escolha da cultivar, época de semeadura, população de plantas, controle de plantas daninhas insetos e doenças, seguiram as recomendações técnicas para a cultura da soja da EMBRAPA (2013).

Os resultados obtidos foram avaliados pelo teste de normalidade de Shapiro e Wilk (1965) a 5\% de probabilidade. Quando diferenças significativas foram detectadas na análise de variância, as médias foram comparadas pelo teste de Tukey a $5 \%$ de probabilidade, sendo que não foi necessária a transformação dos dados. As análises foram executadas com auxílio do Software AgroEstat (BARBOSA; MALDONADO JUNIOR, 2015).

\section{RESULTADO E DISCUSSÃO}

Nas Tabelas 1 e 2 estão apresentados os resultados obtidos para os parâmetros NNODT, MSNNOT, TNPA, APF, MSR e MSPA da cultivar BRS 7380RR submetida a três densidades de semeadura e três tratamentos envolvendo inoculação no sulco, controle e coinoculação no sulco.

Apenas o parâmetro MSPA (Tabela 2), não apresentou diferença para nenhuma fonte de variação da análise de variância. Com relação a APF apenas teve diferença estatística para tipos de tratamentos F(B), sendo na média geral, os tratamentos que envolveram a aplicação da inoculação e coinoculação no sulco de semeadura foram equivalentes estatisticamente entre si e promoveram incrementos na altura de planta no florescimento em relação ao controle não inoculado (Tabela 2).

$\mathrm{Na}$ Tabela 1, foram verificadas diferenças expressivas quanto a nodulação. Na média geral dos três tratamentos testados para a cultivar de soja BRS 7380 RR o uso da densidade de semeadura de 14 sementes $\mathrm{m}^{-1}$ destacou-se incrementando o número de nódulos totais (NNODT) (83, 45 nódulos planta ${ }^{-1}$ ), sendo estatisticamente superior em relação as outras densidades testadas como 32,12 nódulos para 16 e 36,18 nódulos para 18 sementes $\mathrm{m}^{-1}$. Por outro lado, considerando a média geral das densidades testadas, nota-se que e a inoculação foi equivalente estatisticamente a coinoculação no sulco, diferindo-se apenas do controle não inoculado, apresentou o pior valor médio quanto ao número de nódulos que foi de 45,59 nódulos planta ${ }^{-1}$. No desdobramento da interação $\mathrm{F}(\mathrm{C})$ na densidade de 14 sementes $\mathrm{m}^{-1}$ semelhante ao ocorrido na média geral, a coinoculação não diferiu da inoculação sendo equivalentes e superiores ao controle. Entretanto, na densidade de 16 sementes $\mathrm{m}^{-1}$ a inoculação no sulco de semeadura não diferiu estatisticamente do controle, e foi superior estatisticamente a coinoculação. E para a densidade de 18 sementes $\mathrm{m}^{-1}$ a coinoculação e controle foram equivalentes entre si e superiores a inoculação tradicional no sulco. Ainda considerando a nodulação total, observou-se também que nos três tratamentos testados a densidade de 14 plantas $\mathrm{m}^{-1}$ foi a que mais favoreceu esse parâmetro, promovendo maior nodulação total (Tabela1).

A presença de nodulação até mesmo no tratamento controle, ocorreu devido a elevada população microbiana já estabelecida na área. Valendo ressaltar a importância de reinoculação anual com inoculantes biológicos, pelo fato da competição com microorganismos menos eficientes e que competem pelo sítio 
de ação dos nódulos (CARVALHO et al., 2005).

Com relação a massa seca de nódulos total (MSNNOT) no desdobramento da interação nota-se que na densidade de 14 sementes $\mathrm{m}^{-1}$ a inoculação apresentou $297 \mathrm{mg}$ planta ${ }^{-1}$ não diferindo estatisticamente da coinoculação $(314 \mathrm{mg})$, diferindo porém do controle que foi inferior, ou seja, apresentou menor média de massa seca nodular total $\left(181 \mathrm{mg}\right.$ planta $\left.{ }^{-1}\right)$. Por outro lado, na maior densidade de semeadura testada, a coinoculação no sulco mostrou $326 \mathrm{mg}$ planta $^{-1}$ e foi superior a inoculação e controle que não diferiram entre si e apresentaram respectivamente, 129 e $190 \mathrm{mg}_{\text {planta }}{ }^{-1}$ de massa seca nodular. Já, na densidade de 16 plantas $\mathrm{m}^{-1}$, os três tratamentos foram equivalentes estatisticamente entre si. Ainda na Tabela 1, no desdobramento da interação, dentro da coinoculação, portanto as densidades menor e maior testadas incrementaram a MSNNOT. Entretanto, dentro da inoculação a densidade menor de 14 sementes $\mathrm{m}^{-1}$ foi a que mais incrementou este parâmetro em relação as demais densidades testadas. Para o controle, não foram observadas diferenças entre densidades de semeadura quanto a esse parâmetro. Estes resultados corroboram em parte com os obtidos por Gitti et al. (2014) que mencionam que o uso do Azospirillum brasilense em soja, proporcionou maior NNODT e MSNNOT. As médias gerais obtidas no presente trabalho foram de 50,58 nódulos planta $^{-1}$ e $209 \mathrm{mg}$ planta $^{-1}$. Os valores observados estão dentro da faixa recomendada para eficiência simbiótica no início do florescimento que é de 15 a 30 nódulos por planta com 100 a 200 mg de massa seca nodular (HUNGRIA et al., 2001).

Para o teor de nitrogênio acumulado na parte aérea (TNPA) considerando a média geral das densidades testadas, nota-se que para os tratamentos inoculação, controle e coinoculação não foram verificadas diferenças estatísticas. Resultados semelhantes foram obtidos por Zilli et al. (2010) e Bulegon et al. (2016) que também não obtiveram em seus resultados de pesquisa diferenças para acúmulo de nitrogênio na parte aérea. Isso, pode estar relacionado ao fato das plantas ainda estarem utilizando o $\mathrm{N}$ disponível no solo até o período em que foram conduzidas as coletas para avaliação desse parâmetro de FBN (ZILLI, 2010). Por sua vez, considerando a média geral dos tratamentos testados, observa-se para as densidades, diferenças estatísticas significativas. A densidade de semeadura de 14 sementes $\mathrm{m}^{-1}$ apresentou menor valor médio de TNPA de 35,16 g. $\mathrm{kg}^{-1}$. Já, a densidade com maior valor médio de TNPA encontrada foi a que utilizou 16 sementes $\mathrm{m}^{-1}$, com 47,45 g. $\mathrm{kg}^{-1}$, diferindo significativamente das demais densidades testadas. No desdobramento da interação nota-se que na densidade de 16 plantas $\mathrm{m}^{-1} \mathrm{a}$ inoculação no sulco foi superior e diferiu estatisticamente aos demais tratamentos testados. Entretanto, na maior densidade de semeadura, a coinoculação foi superior estatisticamente. Por outro lado, dentro da inoculação no sulco, a densidade de 16 sementes $\mathrm{m}^{-1}$ foi a que melhor incrementou este parâmetro. Por sua vez, na coinoculação, as densidades de 16 e 18 sementes $\mathrm{m}^{-1}$ foram superiores e equivalentes entre si quando comparadas a de 14 sementes $\mathrm{m}^{-1}$ (Tabela 1). 
Tabela 1. Nodulação (número e massa seca nodular total) e teor de nitrogênio na parte aérea avaliados no estádio fenológico R1 na cultivar de soja BRS 7380 RR submetida a três densidades de semeadura e diferentes tratamentos envolvendo inoculação, coinoculação no sulco de semeadura e controle. Tanabi-SP. Ano agrícola 2018-19.

\begin{tabular}{|c|c|c|c|c|c|c|c|c|c|c|c|c|}
\hline \multirow{2}{*}{$\begin{array}{l}\begin{array}{l}\text { Densidade (A) } \\
\text { sementes } \mathbf{~ m}^{-1}\end{array} \\
\text { Tratamentos(B) }\end{array}$} & \multicolumn{3}{|c|}{ 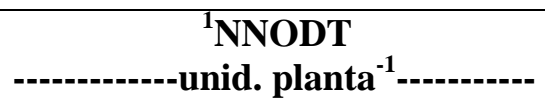 } & \multirow{2}{*}{$\begin{array}{l}\text { Média } \\
\text { Geral } \\
\text { - }\end{array}$} & \multicolumn{3}{|c|}{$\begin{array}{c}{ }^{1} \text { MSNNOT }^{2} \\
-\end{array}$} & \multirow{2}{*}{$\begin{array}{l}\text { Média } \\
\text { Geral } \\
-\end{array}$} & \multicolumn{3}{|c|}{ 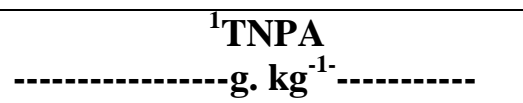 } & \multirow{2}{*}{$\begin{array}{l}\text { Média } \\
\text { Geral } \\
- \\
\end{array}$} \\
\hline & $\mathbf{I}$ & $\mathbf{C}$ & COI & & I & $\mathbf{C}$ & COI & & I & $\mathbf{C}$ & COI & \\
\hline 14 & $95,89 \mathrm{aA}$ & $62,56 \mathrm{aB}$ & $91,89 \mathrm{aA}$ & $83,45 a$ & $297 \mathrm{aA}$ & $181 \mathrm{aB}$ & $314 \mathrm{aA}$ & $264 \mathrm{a}$ & $35,00 \mathrm{cA}$ & $34,53 \mathrm{bA}$ & $35,93 \mathrm{bA}$ & $35,16 \mathrm{c}$ \\
\hline 16 & $37,56 \mathrm{bA}$ & $33,67 \mathrm{bAB}$ & $25,12 \mathrm{cB}$ & $32,12 b$ & $187 \mathrm{bA}$ & $108 \mathrm{aA}$ & $148 \mathrm{bA}$ & $147 \mathrm{~b}$ & $50,63 \mathrm{aA}$ & $46,00 \mathrm{aB}$ & $45,71 \mathrm{aB}$ & $47,45 \mathrm{a}$ \\
\hline 18 & $28,22 \mathrm{bB}$ & $40,55 \mathrm{bA}$ & $39,78 \mathrm{bA}$ & $36,18 b$ & $129 \mathrm{bB}$ & $190 \mathrm{aB}$ & 326 aA & $215 \mathrm{ab}$ & $39,07 \mathrm{bB}$ & $43,20 \mathrm{aAB}$ & $44,20 \mathrm{aA}$ & $42,16 \mathrm{~b}$ \\
\hline $\mathrm{F}(\mathrm{A})$ & \multicolumn{4}{|c|}{$107,01 * *$} & \multicolumn{4}{|c|}{$12,99 *$} & \multicolumn{4}{|c|}{$123,70^{* *}$} \\
\hline $\mathrm{F}(\mathrm{B})$ & \multirow{2}{*}{\multicolumn{4}{|c|}{$\begin{array}{c}7,72 * \\
22,42 * *\end{array}$}} & \multicolumn{4}{|c|}{$56,74 * *$} & \multicolumn{4}{|c|}{$0,18 \mathrm{NS}$} \\
\hline $\mathrm{F}(\mathrm{C})$ & & & & & \multicolumn{4}{|c|}{$5,98 *$} & \multicolumn{4}{|c|}{$7,47 * *$} \\
\hline Média Geral & $53,89 \mathrm{~A}$ & $45,59 \mathrm{~B}$ & $52,26 \mathrm{AB}$ & 50,58 & $204 \mathrm{~B}$ & $160 \mathrm{C}$ & $263 \mathrm{~A}$ & 209 & $41,57 \mathrm{~A}$ & $41,24 \mathrm{~A}$ & $41,95 \mathrm{~A}$ & 41,59 \\
\hline CV\% (A) & 16,36 & & & & 23,33 & & & & 4,00 & & & \\
\hline $\mathrm{CV} \%$ (B) & 9,39 & & & & 9,87 & & & & 6,01 & & & \\
\hline CV\% (C) & 9,72 & & & & 22,69 & & & & 4,20 & & & \\
\hline
\end{tabular}

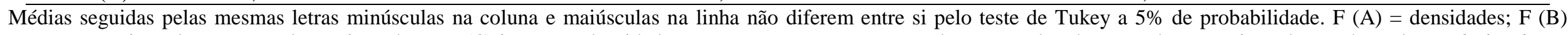

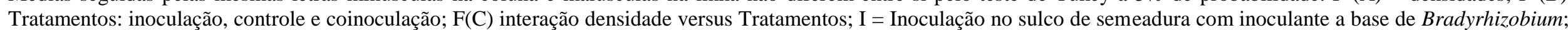

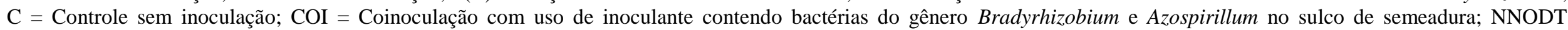

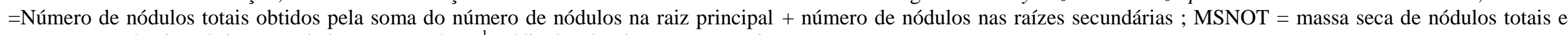
TNPA = teor de nitrogênio acumulado na parte aérea. ${ }^{1}$ Média de três plantas por repetição. 
Tabela 2. Altura de planta no florescimento e massa seca da parte aérea e da raiz avaliados no estádio fenológico R1 na cultivar de soja BRS 7380 RR submetida a três densidades de semeadura e diferentes tratamentos envolvendo inoculação, coinoculação no sulco de semeadura e controle. Tanabi-SP. Ano agrícola 201819.

\begin{tabular}{|c|c|c|c|c|c|c|c|c|c|c|c|c|}
\hline \multirow{2}{*}{ 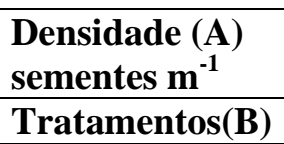 } & \multicolumn{3}{|c|}{$\begin{array}{c}\mathrm{APF}^{1} \\
-\end{array}$} & Média & \multicolumn{3}{|c|}{ 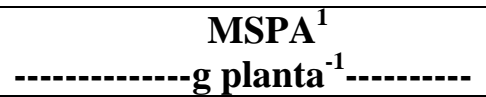 } & \multirow{2}{*}{$\begin{array}{l}\text { Média } \\
\text { Geral } \\
-\end{array}$} & \multicolumn{3}{|c|}{  } & \multirow{2}{*}{$\begin{array}{l}\text { Média } \\
-\end{array}$} \\
\hline & I & $\mathbf{C}$ & COI & - & I & $\mathrm{C}$ & COI & & I & C & COI & \\
\hline 14 & - & - & - & $45,67 \mathrm{a}$ & - & - & - & - & $1,25 \mathrm{aA}$ & $0,92 \mathrm{aA}$ & $1,06 \mathrm{bA}$ & $1,08 \mathrm{~b}$ \\
\hline 16 & - & & - & $46,22 \mathrm{a}$ & - & - & - & - & $0,85 \mathrm{bA}$ & $0,99 \mathrm{aA}$ & $0,97 \mathrm{bA}$ & $0,94 \mathrm{~b}$ \\
\hline 18 & - & - & - & $46,67 \mathrm{a}$ & - & - & - & - & $0,91 \mathrm{abB}$ & $0,90 \mathrm{aB}$ & $2,29 \mathrm{aA}$ & $1,37 \mathrm{a}$ \\
\hline $\mathrm{F}(\mathrm{A})$ & \multicolumn{4}{|c|}{$0,06 \mathrm{NS}$} & \multicolumn{4}{|c|}{$1,25 \mathrm{NS}$} & \multicolumn{4}{|c|}{$51,61 * *$} \\
\hline $\mathrm{F}(\mathrm{B})$ & \multicolumn{4}{|c|}{$12,32^{*}$} & \multicolumn{4}{|c|}{$3,07 \mathrm{NS}$} & \multicolumn{4}{|c|}{$18,68 * *$} \\
\hline $\mathrm{F}(\mathrm{C})$ & \multicolumn{4}{|c|}{$0,76 \mathrm{NS}$} & \multirow{2}{*}{\multicolumn{3}{|c|}{$1,90 \mathrm{NS}$}} & & \multicolumn{4}{|c|}{$19,97 * *$} \\
\hline Média & $47,33 \mathrm{~A}$ & $43,00 \mathrm{~B}$ & $48,22 \mathrm{~A}$ & 46,19 & & & & 8,20 & $1,00 \mathrm{~B}$ & $0,94 \mathrm{~B}$ & $1,44 \mathrm{~A}$ & 1,13 \\
\hline $\mathrm{CV} \%$ (A) & \multicolumn{4}{|c|}{13,76} & \multicolumn{4}{|c|}{9,80} & \multicolumn{4}{|c|}{8,09} \\
\hline $\mathrm{CV} \%$ (B) & \multicolumn{4}{|c|}{5,17} & \multicolumn{4}{|c|}{10,97} & \multicolumn{4}{|c|}{16,83} \\
\hline $\mathrm{CV} \%$ (C) & \multicolumn{4}{|c|}{10,11} & \multicolumn{3}{|c|}{10,55} & & \multicolumn{4}{|c|}{16,40} \\
\hline
\end{tabular}

Médias seguidas pelas mesmas letras minúsculas na coluna e maiúsculas na linha não diferem entre si pelo teste de Tukey a 5\% de probabilidade. $\mathrm{F}$ (A) = densidades; $\mathrm{F}$ (B)

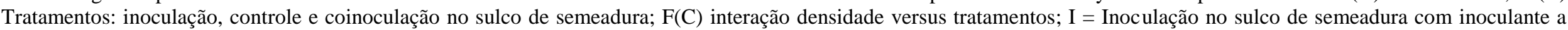

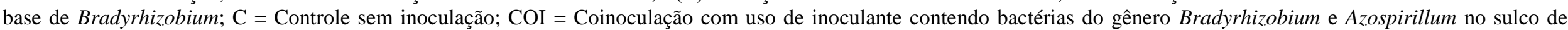
semeadura; APF = Altura de planta no florescimento; MSPA = massa seca da parte aérea e MSR = massa seca da raiz. ${ }^{1}$ Média de 3 plantas por repetição. 
Na tabela 2 para massa seca da raiz (MSR) considerando a média geral das densidades, nota-se que a coinoculação foi superior estatisticamente aos demais tratamentos testados. $\mathrm{O}$ acréscimo verificado com o uso dessa recente prática pode estar associado a capacidade de bactérias do gênero Azospirillum brasilense na produção de fitorhormônios de crescimento vegetal, aliado a maior absorção de agua e nutrientes (HUNGRIA et al., 2013). Em relação a média geral dos tratamentos, a densidade de 18 sementes $\mathrm{m}^{-1}$ foi estatisticamente superior as demais testadas proporcionando maior MSR. No desdobramento da interação, dentro da coinoculação a maior densidade de sementes por metro linear (18) foi responsável pela produção de 2,29 $\mathrm{g} \mathrm{planta}^{-1}$ de MSR quando comparada ao controle não inoculado e inoculação que foram equivalentes entre si, com respectivamente valores médios de MSR de 1,06 g na densidade de 14 sementes e 0,97 g planta $^{-1}$ na densidade de 16 sementes $\mathrm{m}^{-1}$. Dentro da inoculação, as densidades de 14 e 18 sementes $\mathrm{m}^{-1}$ não diferiram entre si e foram superiores estatisticamente a de 16 sementes $\mathrm{m}^{-1}$. E no controle, as densidades não se diferenciaram estatisticamente entre si.

Os caracteres AIV (Tabela 3) e MMG (Tabela 4), não apresentaram diferença para nenhuma fonte de variação da análise de variância. Para massa de mil grãos os resultados obtidos no presente trabalho não corroboram com Val et al. (1971), na qual verificaram que existe variação na massa de sementes com a mudança da densidade de semeadura; e com Weber et al. (1966) que também observaram aumento desse componente relacionado ao aumento da população de plantas. Já para AIV, Santos et al. (2018) também verificaram diferenças significativas e que com o aumento da densidade de semeadura observaram aumento na altura de inserção da primeira vagem.

Com relação a APM apenas teve diferença estatística a 5\% para densidades $\mathrm{F}(\mathrm{A})$ e a $1 \%$ na interação $\mathrm{F}(\mathrm{C})$. Na média geral, a densidade de 14 sementes $\mathrm{m}^{-1}$ destacou-se incrementando a altura de plantas na maturação em relação a densidade de 16 sementes $\mathrm{m}^{-1}$ que foi inferior estatisticamente, apesar de ter igualdade estatística em relação a densidade de 18 sementes $\mathrm{m}^{-1}$. Santos et al. (2018) avaliando as cultivares BRS 1074 IPRO e BRS 1001 IPRO, notaram que a densidade de 18 sementes por metro foi superior em termos de altura da planta que densidades menores como a de 12 a 8 sementes por metro e que possíveis causas estariam relacionadas ao provável estiolamento em busca de maior absorção e aproveitamento de luz, que ocorre em altas populações de plantas. No desdobramento da interação, verificou-se que dentro da inoculação, as densidades 14 e 16 sementes $\mathrm{m}^{-1}$ foram equivalentes estatisticamente e superiores em termos de estatura quando comparadas a densidade de semeadura de 18 sementes $\mathrm{m}^{-1}$. Já, dentro da coinoculação, as densidades maior e menor foram equivalentes e superiores a de 16 sementes $\mathrm{m}^{-1}$. O controle não inoculado não teve variação estatística entre as densidades. Entretanto, considerando a densidade de 14 sementes $\mathrm{m}^{-1}$ nota-se que a inoculação e coinoculação não diferiram entre si mas foram superiores ao controle não inoculado. E na densidade de 16 sementes $\mathrm{m}^{-1}$, a inoculação não diferiu estatisticamente do controle e ambas foram superiores a prática da coinoculação. Na densidade de 18 sementes $\mathrm{m}^{-1}$ não ocorreram diferenças entre os tratamentos testados (Tabela 3).

Ainda na tabela 3, em relação ao estande final obtido (EF) quando se considera a média geral das três densidades testadas, observou-se que a prática da coinoculação avultou-se aumentando o estande final de plantas que teve valor médio de 9,63 plantas $\mathrm{m}^{-1}$ sendo estatisticamente superior aos demais tratamentos testados. Por sua vez, na média geral dos três tratamentos avaliados no presente trabalho, a densidade de semeadura de 14 sementes $\mathrm{m}^{-1}$ foi o destaque, sendo estatisticamente superior as demais densidades analisadas, com 9,33 plantas $\mathrm{m}^{-1}$ finais. No desdobramento da interação, dentro da densidade de semeadura de 14 sementes $\mathrm{m}^{-1}$, a coinoculação foi superior estatisticamente a inoculação e controle que tiveram igualdade estatística. Já, na densidade de 16 sementes $\mathrm{m}^{-1}$, a inoculação foi superior a coinoculação e controle e em relação a de 18 sementes $\mathrm{m}^{-1}$ a coinoculação foi superior estatisticamente a inoculação apesar de não diferir estatisticamente do controle não inoculado. Por outro lado, no 
desdobramento da interação dentro da inoculação e coinoculação nota-se que a densidade de 14 sementes por $\mathrm{m}^{-1}$ sobressaiu-se e foi superior estatisticamente as outras densidades testadas. 
Tabela 3. Estande final, altura de planta e inserção da primeira vagem, avaliados no estádio reprodutivo R8 na cultivar de soja BRS 7380 RR submetida a três densidades de semeadura e diferentes tratamentos envolvendo inoculação, coinoculação no sulco de semeadura e controle. Tanabi-SP. Ano agrícola 2018-19.

\begin{tabular}{|c|c|c|c|c|c|c|c|c|c|c|c|c|}
\hline \multirow{2}{*}{$\begin{array}{l}\begin{array}{l}\text { Densidade (A) } \\
\text { sementes } \mathbf{m}^{-1}\end{array} \\
\text { Tratamentos(B) }\end{array}$} & \multicolumn{3}{|c|}{$\begin{array}{c}\text { EF }^{1} \\
\end{array}$} & \multirow{2}{*}{$\begin{array}{l}\text { Média } \\
\text { Geral } \\
-\end{array}$} & \multicolumn{3}{|c|}{$\begin{array}{l}\mathrm{APM}^{2} \\
-\mathbf{m}-----\end{array}$} & \multirow{2}{*}{$\begin{array}{l}\text { Média } \\
\text { Geral }\end{array}$} & \multicolumn{3}{|c|}{$\begin{array}{c}\mathrm{AIV}^{2} \\
-\end{array}$} & \multirow{2}{*}{$\begin{array}{l}\text { Média } \\
\text { Geral } \\
- \\
\end{array}$} \\
\hline & I & $\mathbf{C}$ & COI & & I & $\mathrm{C}$ & COI & & $\mathbf{I}$ & $\mathrm{C}$ & COI & \\
\hline 14 & $8,30 \mathrm{aB}$ & $7,73 \mathrm{bB}$ & $11,97 \mathrm{aA}$ & $9,33 \mathrm{a}$ & $81,25 \mathrm{aA}$ & $69,00 \mathrm{aB}$ & $72,25 \mathrm{aAB}$ & $74,17 \mathrm{a}$ & - & - & - & $16,44 a$ \\
\hline 16 & $6,73 \mathrm{bB}$ & $9,27 \mathrm{aA}$ & $8,47 \mathrm{bA}$ & $8,16 b$ & $73,33 \mathrm{abA}$ & $68,33 \mathrm{aAB}$ & $61,33 \mathrm{bB}$ & $67,67 \mathrm{~b}$ & - & - & - & $14,88 \mathrm{a}$ \\
\hline 18 & $6,73 \mathrm{bB}$ & $8,40 \mathrm{abA}$ & $8,47 \mathrm{bA}$ & $7,87 \mathrm{~b}$ & $67,50 \mathrm{bA}$ & $72,33 \mathrm{aA}$ & $74,33 \mathrm{aA}$ & 71,39ab & - & - & - & $13,94 \mathrm{a}$ \\
\hline $\mathrm{F}(\mathrm{A})$ & \multicolumn{4}{|c|}{$18,57 * *$} & \multicolumn{4}{|c|}{$7,93 *$} & \multicolumn{4}{|c|}{$2,19 \mathrm{NS}$} \\
\hline $\mathrm{F}(\mathrm{B})$ & \multicolumn{4}{|c|}{$33,55^{* *}$} & \multicolumn{4}{|c|}{$1,40 \mathrm{NS}$} & \multicolumn{4}{|c|}{$1,73 \mathrm{NS}$} \\
\hline $\mathrm{F}(\mathrm{C})$ & \multicolumn{4}{|c|}{$12,34 * *$} & \multicolumn{4}{|c|}{$7,52 * *$} & \multicolumn{4}{|c|}{$1,05 \mathrm{NS}$} \\
\hline Média Geral & $7,26 \mathrm{C}$ & $8,47 \mathrm{~B}$ & $9,63 \mathrm{~A}$ & 8,45 & $74,03 \mathrm{~A}$ & $69,89 \mathrm{~A}$ & $69,31 \mathrm{~A}$ & 71,07 & $14,94 \mathrm{~A}$ & $14,67 \mathrm{~A}$ & $15,67 \mathrm{~A}$ & 15,09 \\
\hline CV\% (A) & \multicolumn{4}{|c|}{6,40} & \multicolumn{4}{|c|}{4,89} & \multicolumn{4}{|c|}{16,96} \\
\hline CV\% (B) & \multicolumn{4}{|c|}{7,29} & \multicolumn{4}{|c|}{9,20} & \multicolumn{4}{|c|}{7,81} \\
\hline $\mathrm{CV} \%(\mathrm{C})$ & \multicolumn{4}{|c|}{7,92} & \multicolumn{3}{|c|}{5,22} & & \multicolumn{4}{|c|}{14,61} \\
\hline
\end{tabular}

Médias seguidas pelas mesmas letras minúsculas na coluna e maiúsculas na linha não diferem entre si pelo teste de Tukey a 5\% de probabilidade. $\mathrm{F}$ (A) = densidades; $\mathrm{F}$ (B) tratamentos utilizados: inoculação, controle e coinoculação; $\mathrm{F}(\mathrm{C})$ interação densidade versus tratamentos utilizados; $\mathrm{I}=\mathrm{Inoculação} \mathrm{no} \mathrm{sulco} \mathrm{de} \mathrm{semeadura} \mathrm{com} \mathrm{inoculante} \mathrm{a} \mathrm{base}$ de Bradyrhizobium; C = Controle sem inoculação; COI = Coinoculação com uso de inoculante contendo bactérias do gênero Bradyrhizobium e Azospirillum no sulco de semeadura; APM = Altura de planta na maturação; AIV = altura de inserção da primeira vagem e EF = estande final. ${ }^{1}$ média de 2 linhas de $5 \mathrm{~m}$ de comprimento; ${ }^{2}$ média de 4 plantas por repetição 
Tabela 4. Massa de mil grãos e produtividade de grãos, avaliados no estádio reprodutivo R8 na cultivar de soja BRS 7380 RR submetida a três densidades de semeadura $\mathrm{m}^{-1}$ e diferentes tratamentos envolvendo inoculação, coinoculação no sulco de semeadura e controle. Tanabi-SP. Ano agrícola 2018-19.

\begin{tabular}{|c|c|c|c|c|c|c|c|c|}
\hline \multirow{2}{*}{ 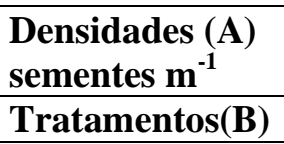 } & \multicolumn{3}{|c|}{ MMG $^{1}$} & \multirow{2}{*}{$\begin{array}{l}\text { Média geral } \\
- \\
\end{array}$} & \multicolumn{3}{|c|}{ 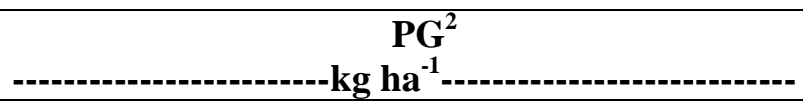 } & \multirow{2}{*}{$\begin{array}{l}\text { Média geral } \\
\text { - } \\
\end{array}$} \\
\hline & I & $\mathbf{C}$ & COI & & I & $\mathbf{C}$ & COI & \\
\hline 14 & - & - & - & $121,99 a$ & $1390,13 \mathrm{cB}$ & $1416,30 \mathrm{bB}$ & $1850,70 \mathrm{bA}$ & $1552,38 \mathrm{c}$ \\
\hline 16 & - & - & - & $128,43 a$ & $2527,36 \mathrm{aA}$ & $2201,20 \mathrm{aB}$ & $2130,90 \mathrm{aB}$ & $2286,48 \mathrm{a}$ \\
\hline 18 & - & - & - & $124,57 \mathrm{a}$ & $1895,54 \mathrm{bC}$ & $2031,29 \mathrm{aB}$ & $2186,96 \mathrm{aA}$ & $2037,93 \mathrm{~b}$ \\
\hline $\mathrm{F}(\mathrm{A})$ & & & $3,80 \mathrm{NS}$ & & \multicolumn{4}{|c|}{$57,58 * *$} \\
\hline $\mathrm{F}(\mathrm{B})$ & & & $1,82 \mathrm{NS}$ & & \multicolumn{4}{|c|}{$20,14 * *$} \\
\hline $\mathrm{F}(\mathrm{C})$ & & & $0,73 \mathrm{NS}$ & & \multicolumn{4}{|c|}{$44,56 * *$} \\
\hline Média geral & $127,88 \mathrm{~A}$ & $124,67 \mathrm{~A}$ & $122,43 \mathrm{~A}$ & 125,00 & $1937,68 \mathrm{~B}$ & $1882,93 \mathrm{~B}$ & $2056,19 \mathrm{~A}$ & 1958,93 \\
\hline CV\% (A) & & & 3,99 & & \multicolumn{4}{|c|}{7,54} \\
\hline CV\% (B) & & & 4,87 & & \multicolumn{4}{|c|}{3,02} \\
\hline $\mathrm{CV} \%(\mathrm{C})$ & & & 5,59 & & \multicolumn{4}{|c|}{3,10} \\
\hline
\end{tabular}

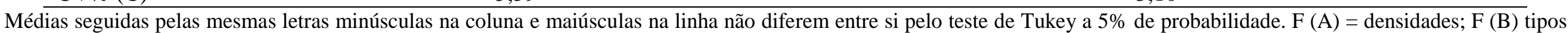
de tratamentos utilizados: inoculação, controle e coinoculação; $\mathrm{F}(\mathrm{C})$ interação densidade versus tratamentos utilizados; $\mathrm{I}=$ Inoculação no sulco de semeadura com inoculante a

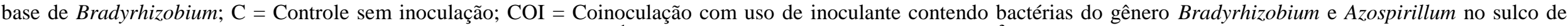
semeadura; MMG=massa de mil grãos $; \mathrm{PG}=$ produtividade de grãos; ${ }^{1}$ média de 2 linhas de $5 \mathrm{~m}$ de comprimento $;{ }^{2}$ média de 4 plantas por repetição. 
$\mathrm{Na}$ Tabela 4 quando se analisa o caráter produtividade de grãos (PG), observa-se na média geral, que a coinoculação foi superior estatisticamente aos demais tratamentos testados, com 2056, $19 \mathrm{~kg} \mathrm{ha}^{-1}$, com incrementos significativos de $118,51 \mathrm{~kg} \mathrm{ha}^{-1}$ quando comparado a inoculação tradicional com somente Bradyrhizobium e 173,26 kg ha ${ }^{-1}$ em relação ao controle não inoculado. Em relação ainda as médias gerais, para densidade de semeadura a de 16 sementes $\mathrm{m}^{-1}$ apresentou uma produtividade de $2286,48 \mathrm{~kg} \mathrm{ha}^{-1}$, estatisticamente superior as demais testadas. No desdobramento da interação, nota-se que dentro da coinoculação e controle as densidades de 16 e 18 sementes $\mathrm{m}^{-1}$ foram equivalentes entre si e superiores quanto a PG quando comparadas a densidade de 14 sementes $\mathrm{m}^{-1}$. Porém, dentro da inoculação, a densidade de 16 sementes $\mathrm{m}^{-1}$ sobressaiu-se estatisticamente entre as demais testadas com maior valor médio de produtividade de grãos encontrado no trabalho que foi de $2527,36 \mathrm{~kg} \mathrm{ha}^{-1}$. Assim, na densidade de 16 sementes $\mathrm{m}^{-1}$ a inoculação com Bradyrhizobium no sulco de semeadura foi estatisticamente superior aos demais, com incremento de $396,46 \mathrm{~kg}$ ha ${ }^{-1}$ quando comparado a coinoculação que teve valor médio de $2130,90 \mathrm{~kg} \mathrm{ha}^{-1}$. Entretanto, nas densidades de 14 e 18 sementes $\mathrm{m}^{-1}$, a coinoculação foi superior a inoculação tradicional, bem como, ao controle não inoculado. De modo geral, os resultados obtidos no presente trabalho foram inferiores ao obtido na safra anterior por Barbaro-Torneli et al. (2018a) que ao avaliarem a influência de modos de aplicação da inoculação e coinoculação no desempenho agronômico da soja em dois locais Riolândia e Pindorama-SP de semeadura, verificaram como média geral do experimento uma PG de 4204, $41 \mathrm{~kg} \mathrm{ha}^{-1}$, sendo que a coinoculação no sulco foi responsável por $4589 \mathrm{~kg}$ ha $^{-1}$ e a inoculação no sulco de $4298 \mathrm{~kg} \mathrm{ha}^{-1}$. Em outra pesquisa realizada por Bárbaro-Torneli et al. (2018b) destinada a avaliação de cultivares de soja no Estado de São Paulo em resposta a aplicação de inoculantes no sulco de semeadura, verificou-se na média de quatro locais de avaliação (Manduri, Araçatuba, Riolândia e Pindorama) que a coinoculação no sulco de semeadura com o uso da mesma formulação da empresa Stoller do Brasil Ltda na dose de $0,45 \mathrm{~L} \mathrm{ha}^{-1}$ proporcionou expressivo aumento em termos de produtividade de grãos da ordem de $271,77 \mathrm{~kg} \mathrm{ha}^{-1}$ ou 4,5 sacas ha ${ }^{-1}$ e 457,55 $\mathrm{kg} \mathrm{ha}^{-1}$ ou 7,62 sacas $\mathrm{ha}^{-1}$, respectivamente, quando comparada a inoculação com somente Bradyrhizobium na dose utilizada de $0,4 \mathrm{~L} \mathrm{ha}^{-1}$, ou seja metade da dose que foi utilizada no presente trabalho $\left(0,8 \mathrm{~L} \mathrm{ha}^{-1}\right)$ e controle não inoculado. Ainda em relação a esses últimos autores, a cultivar BRS 7380 RR que foi também utilizada no presente trabalho média geral dos quatro locais de avaliação e três tratamentos testados (inoculação, controle e coinoculação) mostrou valor médio de $3211,42 \mathrm{~kg} \mathrm{ha}^{-1}$, bem superior ao encontrado no presente trabalho. Possível causa da baixa produtividade em geral do ensaio está relacionada à falta de chuvas no período de enchimento de vagens que afetou a cultivar proporcionando abortamento de flores e vagens e consequentemente o que levou a menor produtividade de grãos (CIIAGRO, 2019).

\section{CONCLUSÃO}

A interação densidade de semeadura versus diferentes tratamentos testados teve efeito nos parâmetros número e massa seca nodular, massa seca da raiz e teor de nitrogênio acumulado na parte aérea, bem como, nos caracteres estande final, altura de planta na maturação e produtividade de grãos.

A maior produtividade de grãos obtida foi na densidade de semeadura de 16 sementes $\mathrm{m}^{-1}$ no tratamento de inoculação tradicional no sulco utilizando 8 doses do inoculante. 


\section{REFERÊNCIAS}

BÁRBARO, I. M.et al. Técnica alternativa: co-inoculação de soja com Azospirillum e Bradyrhizobium visando incremento de produtividade. 2008. Artigo em Hypertexto. Disponível em:

<http://www.infobibos.com/Artigos/2008 4/coinoculacao/index.htm>. Acesso em: 1 abr. 2015.

BÁRBARO, I. M.et al.Produtividade da soja em resposta à inoculação padrão e co-inoculação.

Colloquium Agrariae v. 5, p. 1-7. 2009.

BÁRBARO, I. M.et al. Resultados preliminares da co-inoculação de Azospirillum juntamente com Bradyrhizobium em soja. Apta Regional-Pesquisa e Tecnologia, Colina-SP v. 8, p. 1-6. 2011.

BÁRBARO-TORNELI, Ivana Marino et al. Influência de modos de aplicação da co-inoculação no desempenho agronômico de soja. Nucleus, Ituverava, p. 105-114, jun. 2018a. Edição Especial 2018. $3^{\circ}$ Encontro Técnico sobre as culturas da soja e milho no noroeste paulista . ISSN 19822278. Disponível em: 〈http://nucleus.feituverava.com.br/index.php/nucleus/article/view/3008>. Acesso em: 30 mar 2019. doi:http://dx.doi.org/10.3738/1982.2278.3008.

BÁRBARO-TORNELI, I. M. et al. Avaliação de cultivares de soja no Estado De São Paulo em resposta à aplicação de inoculantes no sulco de semeadura. Nucleus, Ituverava, p. 55-62, jun. 2018b. ISSN 1982-2278. Disponível em:

<http://nucleus.feituverava.com.br/index.php/nucleus/article/view/3001 >. Acesso em: 30 may 2019. doi:http://dx.doi.org/10.3738/1982.2278.3001.

BARBOSA, J. C.; MALDONADO JÚNIOR, W. AgroEstat: sistema para análises estatísticas de ensaios agronômicos. Jaboticabal: Faculdade de Ciências Agrárias e Veterinárias, Unesp.2015.

BATAGLIA, O. C.et al. Métodos de análise química de plantas. IAC -Instituto Agronômico de Campinas. 1978.

BULEGON, L. G.et al. Componentes de produção e produtividade da cultura da soja submetida à inoculação de Bradyrhizobium e Azospirillum. Terra Latinoamericana v. 34, p.169-176. 2016.

CANASAT. Mapeamento da cana via imagens de satélite de observação da terra. Disponível em: <http://www.dsr.inpe.br/canasat/> Acesso em 8/03/2016.

CANOVA, R.et al. Distribuição de sementes por uma semeadora adubadora em função de alterações mecanismo dosador e de diferentes velocidades de deslocamento. Engenharia na Agricultura, v. 15, n. 3, p. 299-306, 2007.

CARVALHO, F. G.; SELBACH, P. A.; BIZARRO, M. J. Eficiência e competitividade de variantes espontâneos isolados de estirpes de Bradyrhizobium spp recomendadas para a cultura da soja (Glycine max) Revista brasileira de ciência do solo, v. 29, p. 883-891. 2005.

CONAB. Companhia nacional de abastecimento. Disponível em:

<http://www.conab.gov.br/OlalaCMS/uploads/arquivos/16_11_11_14_54_21_boletim_graos_novembro_ 2016.pdf> Acesso em 29/09/2018.

EMBRAPA. EMPRESA BRASILEIRA DE PESQUISA AGROPECUÁRIA. Técnologias de produção de soja. Região Central do Brasil. Londrina: Embrapa Soja, 2013. 265 p.

FERLINI, H. A. Co-Inoculación en Soja (Glicyne max) con Bradyrhizobium japonicum y Azospirillum brasilense Articulos Técnicos-Agricultura. 2006. 
FEHR, W. R.; CAVINESS, J. A. Stages of soybean development. Aimes: Iowa State University, 1977. 11p. (Special Report, 80).

GITTI, D. D. C. Inoculação e Co-inoculação na Cultura da Soja. Tecnologia \& Produção Soja, 2014.

HUNGRIA, M.; CAMPO, R. J.; MENDES, I. D. C. Fixação biológica do nitrogênio na cultura da soja. Embrapa Soja; Brasília, DF: Embrapa Cerrados.2001.

HUNGRIA, M.; NOGUEIRA, M. A.; ARAUJO, R. S. Co-inoculation of soybeans and common beans with rhizobia and azospirilla: strategies to improve sustainability. Biology and Fertility of Soils. v. 49, p.791-801. 2013.

KOPPEN, W. Climatologia. In: PEREIRA, A.R.; ANGELOCCI, L.R.; SENTELHAS, P.C. (Eds), Agrometeorologia. Fundamentos e aplicações práticas. Guaíba: Agropecuária. 478 p. 2001.

PROCÓPIO, S. O.et al. Plantio cruzado na cultura da soja utilizando uma cultivar de hábito de crescimento indeterminado. Revista de Ciências Agrárias, v. 56, p. 319-325, 2013.

RAMBO, L.et al. Rendimento de grãos de soja em função do arranjo de plantas. Ciência Rural, v.33, n.3, p. 405-411, 2003. Disponível em http://www.scielo.br/pdf/cr/v33n3/a03v33n3.df

SANTOS, G. X. L.et al. Efeito da densidade de plantas nas características agronômicas de dois genótipos de soja no noroeste paulista. Nucleus, Edição Especial 2018. $3^{\circ}$ Encontro Técnico sobre as culturas da soja e milho no noroeste paulista. p. 115-124. 2018.

SHAPIRO, S. S.; WILK, M. B. An analysis of variance test for normality: complete samples. Biometrika v. 52, p. 591-611. 1965.

SOUZA C. A.et al. Relação entre densidade de plantas e genótipos de soja Roundup ReadyTM. Planta Daninha, vol. 28, n. 4, p. 887-896, 2010.

VAL, W. M. C. et al. Efeito do espaçamento entre fileiras e da densidade na fileira sobre a produção de grãos e outras características agronômicas da soja. Experimentiae, v.12, n. 12, p. 431-475, 1971.

WEBER, C. R.; SHIBLES, R. M.; BYTH, D. E. Effect of plant population and row spacing on soybean development and production. Agronomy Journal, v. 58, n. 1, p. 99-102, 1996.

ZILLI, J. E.; CAMPO, R. J; HUNGRIA, M. Eficácia da inoculação de Bradyrhizobium em présemeadura da soja. Embrapa Soja- Artigo em periódico indexado (ALICE). 2010.

ZILLI, J. E.et al. Avaliação da fixação biológica de nitrogênio na soja em áreas de primeiro cultivo no cerrado de Roraima. Embrapa Soja-Comunicado Técnico (INFOTECA-E). 9 p. 2006.

ZUFFO, A. M.et al. Morphoagronomic and productive traits of RR soybean due to inoculation via Azospirillum brasilense groove. African Journal of Microbiology Research, v. 10, n. 13, p. 438-444. 2016. 